\title{
COMPLEX TAUBERIAN THEOREMS FOR POWER SERIES $\left({ }^{1}\right)$
}

BY

\section{DIETER GAIER}

1. Introduction. Given a power series $\left({ }^{2}\right)$

$$
f(z)=\sum a_{n} z^{n}=(1-z) \sum s_{n} z^{n}
$$

with radius of convergence 1 , we wish to study the relations between the sequence $(s)$ and its associated function $f(z)$ which may be considered as a transformation $T(s)$. Direct (or Abelian) theorems conclude from the sequence $(s)$ to the transformation $T(s)$, while Tauberian theorems infer conclusions from the behavior of $T(s)$ to the behavior of $(s)$ under specified additional conditions (Tauberian conditions).

For the special transformation $T$ which transforms $(s)$ into $f(z)$ according to (1.1) we distinguish two types of Tauberian theorems. Tauberian theorems of real character use assumptions about $f(z)$ where $z$ is on the real axis, and have real Tauberian conditions; for example

$$
\lim _{z \rightarrow 1-0} f(z)=s \quad \text { and } \quad a_{n} \geqq 0 \text { implies } \lim _{n \rightarrow \infty} s_{n}=s .
$$

In this paper we are concerned with Tauberian theorems of complex character in which the assumptions on $f(z)$ are essentially complex.

One of these complex Tauberian theorems was given first by Fatou [3, p. 389]:

ThEOREM A. If the function $f(z)$ defined by (1.1) is regular at $z=1$ and $a_{n} \rightarrow 0(n \rightarrow \infty)$, then $\sum a_{n}$ converges.

Another theorem of this type is due to M. Riesz (see, e.g. [9, p. 64]):

THEOREM B. If the function $f(z)$ defined by (1.1) is regular in the region

$$
S_{1}:\left\{\begin{array}{lr}
|z|<R, & R>1, \\
|\operatorname{arc}(z-1)|>\theta_{0}, & 0<\theta_{0}<\pi / 2,
\end{array}\right.
$$

and continuous in $\bar{S}_{1}$ (the closure of $S_{1}$ ), then $\sum a_{n}$ converges.

After recalling some well known facts in summability theory we shall relax the assumptions on $f(z)$ in the Theorems $\mathrm{A}$ and $\mathrm{B}$ and prove a theorem which contains both as special cases. The condition about the behavior of

Received by the editors August 7, 1952 .

(1) The results of this paper are contained in the author's Ph.D. Thesis [4]. Numbers in brackets refer to the bibliography at the end of the paper.

(2) We shall always let $\sum_{n=0}^{\infty} a_{n}=\sum a_{n}$. 
$f(z)$ in $S_{1}$ is thereby localized to a smaller region $S_{2}$ in the neighborhood of $z=1$ which is the smallest possible in a certain sense. In $\$ 4$ other regions are taken instead of $S_{2}$.

In $\$ \$ 5$ and 6 we assume that $f(z)=\sum a_{n} z^{n}$ has a positive radius of convergence and that $z=1$ lies on the boundary of the region of $V$-summability associated with $f(z)$. In analogy to the above theorems we infer conclusions from the behavior of $f(z)$ in the neighborhood of $z=1$ to the behavior of $V(s)$. In an extended sense these theorems are also complex Tauberian theorems. For $V$ the methods of Euler-Knopp, Borel, and Meyer-König are taken and thus extensions of results of Karamata, Meyer-König, and Obrechkoff are obtained.

2. The methods $E_{p}, B, S_{\alpha}$. The methods of Euler-Knopp, Borel, and Meyer-König are useful for the analytic continuation of power series. Assume that a series $\sum a_{n}$ with partial sums $s_{n}=\sum_{\nu=0}^{n} a_{\nu}$ is given and that the associated power series $f(z)=\sum a_{n} z^{n}$ has a positive radius of convergence.

a. The method $E_{p}$ (Euler-Knopp) with fixed parameter $p(0<p<\infty)$ is defined by the triangular matrix $\left(E_{p}\right)$ with elements

$$
c_{n \nu}=\frac{1}{2^{p n}}\left(\begin{array}{l}
n \\
\nu
\end{array}\right)\left(2^{p}-1\right)^{n-\nu} \quad(n, \nu=0,1,2, \cdots ; \nu \leqq n) .
$$

The $E_{p}$-transformation of the sequence $s_{n}$ is therefore the new sequence

$$
E_{p}\left(n ; s_{\nu}\right)=\frac{1}{2^{p n}} \sum_{\nu=0}^{n}\left(\begin{array}{l}
n \\
\nu
\end{array}\right)\left(2^{p}-1\right)^{n-\nu} s_{\nu} \quad(n=0,1, \cdots)
$$

and we say that $E_{p^{-}} \lim s_{n}=s\left(\right.$ or $\left.E_{p^{-}} \sum a_{n}=s\right)$ if $\lim _{n \rightarrow \infty} E_{p}\left(n ; s_{\nu}\right)=s$.

For our purposes another definition of the $E_{p}$-transformation of the sequence $s_{n}$ is more suitable. Letting

$$
z=\phi_{p}(w)=\frac{w}{2^{p}-\left(2^{p}-1\right) w}
$$

and developing $f(z)$ into powers of $w$ we obtain a power series

$$
F(w)=\sum a_{n}^{\prime} w^{n}
$$

which is convergent in the neighborhood of $w=0$. It is easy to prove that

$$
E_{p}\left(n ; s_{\nu}\right)=\sum_{\kappa=0}^{n} a_{\kappa}^{\prime} \text { where }\left\{\begin{array}{l}
a_{n}^{\prime}=2^{-p} E_{p}\left(n-1 ; a_{\nu+1}\right)(n=1,2, \cdots), \\
a_{0}^{\prime}=a_{0} .
\end{array}\right.
$$

Hence $E_{p^{-}} \sum a_{n}$ exists if and only if $\sum a_{n}^{\prime}$ converges, and $E_{p^{-}} \lim a_{n}=0$ is necessary for the existence of $E_{p^{-}} \sum a_{n}$. (Here we use the fact that $E_{p}\left(n ; a_{\nu+1}\right)$ $\rightarrow 0(n \rightarrow \infty)$ is equivalent to $E_{p}\left(n ; a_{v}\right) \rightarrow 0(n \rightarrow \infty)$.)

If the method $E_{p}$ is applied to $\sum a_{n} z^{n}$ for different values of $z$, one obtains 
a region $\mathbb{S}_{E_{p}}$ in the $z$-plane in the interior of which the power series is $E_{p}$-summable to the value $f(z)$, whereas it is certainly not summable for any $z$ which lies outside of $\overline{\mathfrak{G}_{E_{p}}}$. On the boundary of $\mathfrak{G}_{E_{p}}$ no general statement can be made and the situation is somewhat similar to the situation on the boundary of the circle of convergence of a power series. $\mathcal{B}_{E_{p}}$ can be constructed from the singularities of $f(z)$; we shall use that $f(z)$ is necessarily regular in $\Omega_{E_{p}}$ $=\Re\left(\left(2^{p}-1\right) /\left(2^{p+1}-1\right)\right)$ if $z=1$ is on the boundary of $\mathfrak{S}_{E_{p}}$. (We denote by $\Re(a)$ the region $|z-a|<1-a$ for $0<a<1$.) $\Omega_{E_{p}}$ is the map of $|w|<1$ under the transformation $z=\phi_{p}(w)$.

b. Two methods of Borel are known. In the case of the "exponential method" we let

$$
B\left(x ; s_{\nu}\right)=e^{-x} \sum \frac{s_{n} x^{n}}{n !}
$$

where the sum exists for $x \geqq 0$ since $\sum a_{n} z^{n}$ has a positive radius of convergence; we say that $B$-lim $s_{n}=s\left(\right.$ or $\left.B-\sum a_{n}=s\right)$ if $\lim _{x \rightarrow \infty} B\left(x ; s_{\nu}\right)=s$.

The second method is called Borel's "integral method" and is often more suitable to power series. With the given series $\sum a_{n}$ we associate the function $\phi(t)=\sum a_{n} t^{n} / n !$ for $t \geqq 0$ and let

$$
B^{\prime}\left(x ; s_{v}\right)=\int_{0}^{x} e^{-t} \phi(t) d t
$$

we say that $B^{\prime}-\lim s_{n}=s$ (or $B^{\prime}-\sum a_{n}=s$ ) if $\lim _{x \rightarrow \infty} B^{\prime}\left(x ; s_{v}\right)=s$.

Concerning the relations between these two methods it is known that

$$
B \text { - } \lim s_{n}=s \text { implies } B^{\prime}-\lim s_{n}=s,
$$

but not conversely. However, since

$$
B\left(x ; s_{\nu}\right)=B\left(x ; a_{v}\right)+B^{\prime}\left(x ; s_{\nu}\right),
$$

the converse of (2.4) is true if and only if $B\left(x ; a_{v}\right) \rightarrow 0(x \rightarrow \infty)$. The relations (2.4) and (2.5) also imply that $B$-lim $a_{n}=0$ is necessary for the existence of $B-\sum a_{n}$.

The region $\Theta_{B}$ of Borel-summability of the power series $\sum a_{n} z^{n}$ is defined analogously to $\mathbb{S}_{E_{p}}$ in a, and is the same for both methods $B$ and $B^{\prime}$. If $z=1$ lies on the boundary of $\mathcal{B}_{B}$, then $f(z)$ is necessarily regular in $\Omega_{B}=\Re(1 / 2)$.

c. The succession of the methods $E_{p}$ and $B$ is continued in a certain sense by the method of Meyer-König [10, p. 272]). This method depends on a parameter $\alpha(0<\alpha<1)$ and is defined by the matrix $\left(S_{\alpha}\right)$ with elements

$$
c_{n \nu}=(1-\alpha)^{n+1}\left(\begin{array}{c}
n+\nu \\
n
\end{array}\right) \alpha^{\nu} \quad(n, \nu=0,1, \cdots) .
$$

The $S_{\alpha}$-transformation of the sequence $s_{n}$ 


$$
S_{\alpha}\left(n ; s_{\nu}\right)=(1-\alpha)^{n+1} \sum\left(\begin{array}{c}
n+\nu \\
n
\end{array}\right) \alpha^{\nu} s_{\nu} \quad(n=0,1, \cdots)
$$

exists if and only if $s_{\nu}=O\left(\nu^{-n} \alpha^{-\nu}\right)$ for $\nu \rightarrow \infty$ and all fixed $n=0,1, \cdots$; one then says that $\left(S_{\alpha}\right)$ is applicable to the sequence $s_{n}$. This obviously implies the regularity of $f(z)$ in $|z|<\alpha$, and the regularity of $f(z)$ in $|z| \leqq \alpha$ is sufficient for the applicability of $\left(S_{\alpha}\right)$ to the sequence $s_{n}$.

Later we use an alternative definition of the $S_{\alpha}$-transformation. Assume that $\left(S_{\alpha}\right)$ is applicable to the sequence $s_{n}$ so that $f(z)$ is regular in $|z|<\alpha$; assume in addition that $f(z)$ is regular at $z=\alpha$. Letting

$$
z=\frac{\alpha}{1-(1-\alpha) w}
$$

and developing $f(z)$ into powers of $w$ we obtain a power series

$$
F(w)=\sum a_{n}^{\prime} w^{n}
$$

which is convergent in the neighborhood of $w=0$. One finds that

$$
S_{\alpha}\left(n ; s_{\nu}\right)=\sum_{\kappa=0}^{n} a_{\kappa}^{\prime} \text { where }\left\{\begin{array}{l}
a_{n}^{\prime}=\frac{\alpha}{1-\alpha} S_{\alpha}\left(n ; a_{\nu+1}\right) \quad(n=1,2, \cdots), \\
a_{0}^{\prime}=a_{0}+\frac{\alpha}{1-\alpha} S_{\alpha}\left(0 ; a_{\nu+1}\right) .
\end{array}\right.
$$

Hence $S_{\alpha^{-}} \sum a_{n}$ exists if and only if $\sum a_{n}^{\prime}$ converges, and $S_{\alpha^{-}} \lim a_{n}=0$ is necessary for the existence of $S_{\alpha^{-}} \sum a_{n}$.

In the application to power series the situation here is slightly more complicated than in a and $\mathrm{b}$. The region of $S_{\alpha}$-summability has not been investigated in full but it is known that the regularity of $f(z)$ in $\Re_{S_{\alpha}}$ $=\Re(1 /(2-\alpha))$ is necessary for the existence of $S_{\alpha^{-}} \sum a_{n}$ (again assuming the regularity of $f(z)$ at $z=\alpha)$.

3. On two theorems of Fatou and M. Riesz. The starting point for our investigations on the circle of convergence of the power series

$$
f(z)=\sum a_{n} z^{n} \quad\left(\limsup _{n \rightarrow \infty}\left|a_{n}\right|^{1 / n}=1\right)
$$

is the Theorems A and B of the introduction. Szász showed [1, pp. 485-486] that in Theorem B the region $S_{1}$ can be replaced by another region which has a finite number of corners on $|z|=1$ instead of one as in the case of $S_{1}\left({ }^{3}\right)$. We show in Theorem 1 that the assumptions on $f(z)$ can be localized completely to a neighborhood of $z=1$ if the condition $a_{n} \rightarrow 0(n \rightarrow \infty)$ is added.

Let $S_{2}$ be the region

(3) Such a region can be expressed by $\bigcap_{1}^{n}\left\{z_{\kappa} \cdot S_{1}\right\}$ with $\left|z_{\kappa}\right|=1(\kappa=1,2, \cdots, n)$. 


$$
S_{2}=S_{2}\left(\delta_{0}, \theta_{0}\right):\left\{\begin{array}{lr}
|z-1|<\delta_{0}, & \delta_{0}>0, \\
|\operatorname{arc}(z-1)|>\theta_{0}, & 0<\theta_{0}<\pi / 2 .
\end{array}\right.
$$

THEOREM 1. Let (3.1) be regular and bounded in $S_{2}$, and $a_{n} \rightarrow 0(n \rightarrow \infty)$. Then $\sum a_{n}=s$ if $A-\sum a_{n}=s$, i.e. if $\lim _{z \rightarrow 1-0} f(z)=s$.

Remarks. a. Theorem 1 contains the Theorems A and B and also Szász's extension of Theorem B. The conditions $a_{n} \rightarrow 0(n \rightarrow \infty)$ and $A-\sum a_{n}=s$ are both necessary for $\sum a_{n}=s$, together they are sufficient for $\sum a_{n}=s$ if $f(z)$ fulfills the above conditions.

b. Let $K=\left(e^{i \phi_{0}}, e^{-i \phi_{0}}\right)$ be a closed subarc of $|z|=1$ within $|z-1|<\delta_{0}$. Under the assumptions of Theorem 1 the series $\sum a_{n} z^{n}$ converges for $z=1$ and Theorem A assures its convergence for every other $z$ on $K$. We extend Theorem 1 by proving the uniform convergence of (3.1) on $K$.

THEOREM 2. Under the assumptions of Theorem 1 the series (3.1) converges uniformly on $K$.

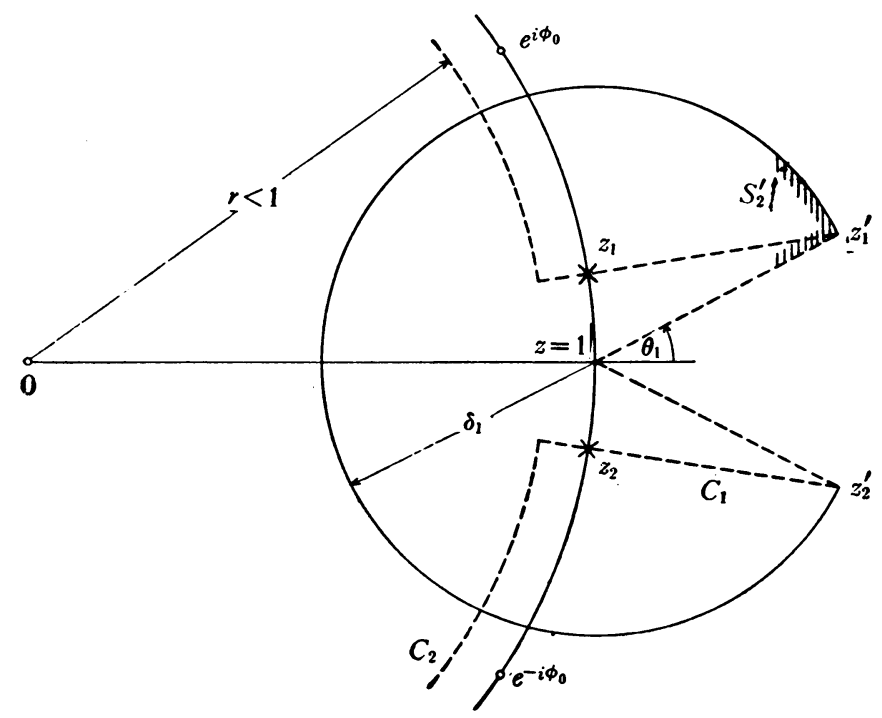

FIG. 1

Proof. By a theorem on bounded functions (see for example $[13$, p. 65]) one obtains first that $f(z)$ is continuous in $\overline{S_{2}^{\prime}}$ where $S_{2}^{\prime}=S_{2}\left(\delta_{1}, \theta_{1}\right)$ for every $\delta_{1}, \theta_{1}$ with $0<\delta_{1}<\delta_{0}$ and $\theta_{0}<\theta_{1}<\pi / 2$. We may assume that $\lim f(z)=0$ for $z \rightarrow 1$ in $S_{2}^{\prime}$ and that $\delta_{1}$ is chosen such that

$$
|f(z)|<\epsilon \cdot\left(\pi \cos \theta_{1}\right) / 8
$$

for $z \in S_{2}^{\prime}$ and a given $\epsilon>0$. Draw the path $C=C_{1}+C_{2}$ as indicated in Fig. 1 . $\left(C_{2}\right.$ is the part of $C$ in $|z|<1$.) 
To prove the uniform convergence of $\sum a_{n} e^{i n \phi}$ for $|\phi| \leqq \phi_{0}$, take first all $\phi$ with $|\phi| \leqq \phi_{1}=(1 / 2)$ arc $z_{1}$. If $\sigma_{n}(\phi)$ are the arithmetic means of $s_{n}(\phi)$ $=\sum_{\nu=0}^{n} a_{\nu} e^{i \nu \phi}$, then

$$
\begin{aligned}
\left|s_{n}(\phi)-\sigma_{n}(\phi)\right|=\frac{1}{n+1}\left|\sum_{\nu=1}^{n} a_{\nu} \nu e^{i \nu \phi}\right|= & \frac{1}{n+1} \mid \frac{1}{2 \pi i}\left\{\sum_{\nu=1}^{n} \nu e^{i \nu \phi} \int_{C_{1}} \frac{f(z) d z}{z^{\nu+1}}\right. \\
& \left.+\int_{C_{2}} f(z) \sum_{\nu=1}^{n} \frac{\nu e^{i \nu \phi}}{z^{\nu+1}} d z\right\} \mid \\
= & \frac{1}{2 \pi(n+1)}\left|A_{n}^{(1)}(\phi)+A_{n}^{(2)}(\phi)\right| .
\end{aligned}
$$

Estimation of $A_{n}^{(1)}$ : For $\nu=1,2, \cdots$

$$
\begin{aligned}
\left|\int_{1}^{z_{1}{ }^{\prime}} \frac{f(z) d z}{z^{\nu+1}}\right| & <\epsilon \cdot \frac{\pi \cos \theta_{1}}{8} \int_{0} \frac{d \tau}{\mid 1+\tau e^{i \theta_{1} \mid \nu+1}} \\
& <\epsilon \cdot \frac{\pi \cos \theta_{1}}{8} \int_{0}^{\infty} \frac{d \tau}{\left(1+\tau \cos \theta_{1}\right)^{\nu+1}}=\frac{\pi}{8 \nu} \cdot \epsilon,
\end{aligned}
$$

and similarly for the other parts of $C_{\mathbf{1}}$. Hence

$$
\left|A_{n}^{(1)}(\phi)\right|<\frac{n \pi}{2} \cdot \epsilon \quad(n=1,2, \cdots),
$$

uniformly for $|\phi| \leqq \phi_{1}$.

Estimation of $A_{n}^{(2)}$ : Evaluating first the finite sum occurring in the expression for $A_{n}^{(2)}$ we obtain

$$
A_{n}^{(2)}(\phi)=e^{i \phi} \int_{C_{2}} f(z) \frac{z^{n+1}-(n+1) z e^{i n \phi}+n e^{i \phi(n+1)}}{z^{n+1}\left(z-e^{i \phi}\right)^{2}} d z .
$$

In this integral we have first

$$
\int_{C_{2}} \frac{f(z) d z}{\left(z-e^{i \phi}\right)^{2}}=o(n)
$$

and we shall prove that also

$$
n \int_{C_{2}} \frac{f(z) d z}{z^{n}\left(z-e^{i \phi}\right)^{2}}=o(n),
$$

always uniformly for $|\phi| \leqq \phi_{1}$. Assume for the moment that (3.4) is true. Then

$$
\left|A_{n}^{(2)}(\phi)\right|<\frac{n \pi}{2} \cdot \epsilon \quad\left(n>n_{1}\right),
$$

uniformly for $|\phi| \leqq \phi_{1}$, and therefore $\left|s_{n}(\phi)-\sigma_{n}(\phi)\right|<\epsilon / 2$ for $n>n_{1}$ and 
$|\phi| \leqq \phi_{1}$. Using now the fact [15, pp. 94-95] that $\sigma_{n}(\phi)$ converges uniformly to $f\left(e^{i \phi}\right)$ for $|\phi| \leqq \phi_{1}$, we get

$$
\left|s_{n}(\phi)-f\left(e^{i \phi}\right)\right| \leqq\left|s_{n}(\phi)-\sigma_{n}(\phi)\right|+\left|\sigma_{n}(\phi)-f\left(e^{i \phi}\right)\right|<\epsilon
$$

for $n>n_{2}$ and all $\phi$ in $|\phi| \leqq \phi_{1}$. Since for $\phi$ with $\phi_{1} \leqq|\phi| \leqq \phi_{0}$ the uniform convergence of $\sum a_{n} e^{i n \phi}$ follows from an extension of Theorem A due to M. Riesz $[15$, p. 90], the proof of Theorem 2 is completed provided (3.4) is true.

For the proof of (3.4) we apply a method of M. Riesz [15], choosing the numbers $b_{1}=b_{1}(\phi)$ and $b_{2}=b_{2}(\phi)$ such that $H\left(z_{1}\right)=H\left(z_{2}\right)=0$ for

$$
H(z)=H(z, \phi)=\frac{1}{\left(z-e^{i \phi}\right)^{2}}+b_{1}+b_{2} z .
$$

One finds that $b_{1}(\phi)$ and $b_{2}(\phi)$ are bounded for $|\phi| \leqq \phi_{1}$. Therefore using (3.3) and $a_{n} \rightarrow 0(n \rightarrow \infty)$ we obtain

$$
\begin{aligned}
\int_{C_{2}} \frac{f(z) H(z) d z}{z^{n}} & =\int_{C_{2}} \frac{f(z) d z}{z^{n}\left(z-e^{i \phi}\right)^{2}}+b_{1} \int_{C} \frac{f(z) d z}{z^{n}}+b_{2} \int_{C} \frac{f(z) d z}{z^{n-1}} \\
& -b_{1} \int_{C_{1}} \frac{f(z) d z}{z^{n}}-b_{2} \int_{C_{1}} \frac{f(z) d z}{z^{n-1}} \\
= & \int_{C_{2}} \frac{f(z) d z}{z^{n}\left(z-e^{i \phi}\right)^{2}}+o(1),
\end{aligned}
$$

so that it remains now to show that

$$
\lim _{n \rightarrow \infty} \int_{C_{2}} \frac{f(z) H(z) d z}{z^{n}}=0,
$$

uniformly for $|\phi| \leqq \phi_{1}$. But by Theorem A the series $\sum a_{n} z^{n}$ converges for $z_{1}$ and $z_{2}$ and therefore uniformly on $C_{2}$ so that

$$
\int_{C_{2}} \frac{f(z) H(z) d z}{z^{n}}=\sum_{\nu} a_{\nu} \int_{C_{2}} z^{\nu-n} H(z) d z
$$

which is a matrix transformation of the zero-sequence $a_{v}$. We have to show

$$
\lim _{n \rightarrow \infty} c_{n \nu}=0 \quad(\nu=0,1, \cdots, \text { fixed }),
$$

$$
\limsup _{n \rightarrow \infty} \sum_{\nu}\left|c_{n \nu}\right|<\infty,
$$

uniformly for $|\phi| \leqq \phi_{1}$, where

$$
c_{n \nu}=c_{n \nu}(\phi)=\int_{C_{2}} z^{\nu-n} H(z, \phi) d z
$$


Integrating twice by parts we get for $\nu \neq n-1, \nu \neq n-2$

$c_{n \nu}=-\frac{1}{\nu-n+1}\left\{\left.\frac{z^{\nu-n+2}}{\nu-n+2} \frac{\partial H(z)}{\partial z}\right|_{z_{1}} ^{z_{2}}-\frac{1}{\nu-n+2} \int_{C_{2}} z^{\nu-n+2} \frac{\partial^{2} H(z)}{\partial z^{2}} d z\right\}$.

Since $H(z)=H(z, \phi)$ is regular in the finite $z$-plane except for $z=e^{i \phi}$ where $|\phi| \leqq \phi_{1}$, we can replace the path $C_{2}$ by the arc: $|\phi| \geqq \operatorname{arc} z_{1}$ of the unit circle. For $z$ on this arc the function $H(z)$ and its first and second derivatives with respect to $z$ are bounded, uniformly for $|\phi| \leqq \phi_{1}$, by a constant $L_{1}$, say. Therefore

$$
\left|c_{n \nu}\right| \leqq L_{1} \frac{2+2 \pi}{(\nu-n+1)(\nu-n+2)} \quad(\nu \neq n-1 ; \nu \neq n-2),
$$

uniformly for $|\phi| \leqq \phi_{1}$, which proves (3.7). But obviously $c_{n, n-1}$ and $c_{n, n-2}$ are uniformly bounded for $|\phi| \leqq \phi_{1}$, by a constant $L_{2}$, say, so that

$$
\begin{aligned}
\sum_{\nu}\left|c_{n \nu}\right| & \leqq 2 L_{2}+L_{1} \sum_{\substack{\nu \neq n-1, \nu \neq n-2 \\
(\nu-n+1)(\nu-n+2)}} \frac{2+2 \pi}{\left(L_{2}+2 L_{1}(2+2 \pi)\right.}
\end{aligned}
$$

which proves (3.8) and therefore completes the proof of Theorems 1 and 2.

4. Weaker assumptions on $f(z)$. Until now our assumptions on $f(z)$ concerned its behavior in the regions $S_{1}$ and $S_{2}$, whose boundaries have an osculation of first order with $|z|=1$ at $z=1$. The question arises what can be said in the case that $S_{1}$ and $S_{2}$ are substituted by the regions $S_{3}$ and $S_{4}$, whose boundaries have an osculation of second order with $|z|=1$ at $z=1$ :

$S_{3}:\left\{\begin{array}{ll}|z|<R, & R>1 \\ |z|<1+c_{0} \phi^{2}, & c_{0}>0\end{array} ; \quad S_{4}:\left\{\begin{array}{l}|z-1|<\delta_{0}, \quad \delta_{0}>0 \\ |z|<1+c_{0} \phi^{2}, c_{0}>0\end{array}\left(z=|z| \cdot e^{i \phi}\right)\right.\right.$.

In the neighborhood of $z=1$ the boundaries of $S_{3}$ and $S_{4}$ behave like a circle which touches $|z|=1$ exteriorly at $z=1$.

First we remark that it is impossible to substitute $S_{1}$ by $S_{3}$ in Theorem B or $S_{2}$ by $S_{4}$ in Theorem 1. For there exist power series which define a function $f(z)$ regular in $S_{3}$ and continuous in $\overline{S_{3}}$ and which are not even $V$-summable for $z=1[5$, p. 331]; by $V$ we denote in this paragraph any of the methods $E_{p}(0<p<\infty), B, S_{\alpha}(0<\alpha<1)$. We shall show, however, that the following modifications of Theorems $B$ and 1 are true.

THEOREM 3. If (3.1) is regular in $S_{3}$ and continuous in $\overline{S_{3}}$, then $\sum a_{n}$ converges provided that $V-\sum a_{n}$ exists $\left.{ }^{4}\right)$.

This is a $V \rightarrow K$-Theorem (" $K$ " standing for "convergence") under complex Tauberian conditions.

( ${ }^{4}$ Theorem 3 and its proof also hold if "continuous in $\overline{S_{3}}$ " is replaced by "bounded in $S_{3} . "$ 
Proof. The assumptions on $f(z)$ imply $a_{n}=O\left(1 / n^{1 / 2}\right)$ [5, p. 327], which is a Tauberian condition for $V$-summability.

We now prove the localization of Theorem 3.

TheOREM 4. Let (3.1) be regular and bounded in $S_{4}$, and $a_{n} \rightarrow 0(n \rightarrow \infty)$. Then $\sum a_{n}=s$ if $V-\sum a_{n}=s\left(^{(5)}\right.$.

Proof. It is sufficient to show

$$
\lim _{n \rightarrow \infty}^{n+\left[e_{n} n \sum_{\nu=n}^{1 / 2]}\right.} a_{\nu}=0
$$

for any positive zero-sequence $\epsilon_{n}$,

because this is a Tauberian condition for $V$-summability. (For $E_{p}$ and $B$ see for example [6, p. 312]; for $S_{\alpha}$ see [4, p. 36].) To prove (4.1) we construct a path similar to the one in Fig. 1, except that the part $\left(z_{2}^{\prime}, 1, z_{1}^{\prime}\right)$ of $C_{1}$ is substituted by a segment of $|z|=1+c_{1} \phi^{2}\left(0<c_{1}<c_{0}\right)$; the parts $\left(z_{2}, z_{2}^{\prime}\right)$ and $\left(z_{1}, z_{1}^{\prime}\right)$ remain rectilinear as in Fig. 1 . Then with $k=\left[\epsilon_{n} n^{1 / 2}\right]$ we have

$$
\sum_{\nu=n}^{n+k} a_{\nu}=\frac{1}{2 \pi i}\left[\sum_{\nu=n}^{n+k} \int_{C_{1}} \frac{f(z) d z}{z^{\nu+1}}+\int_{C_{2}} f(z) \sum_{\nu=n}^{n+k} z^{-\nu-1} d z\right] .
$$

As proved in [5], $\int_{C_{1}} f(z) d z / z^{\nu+1}=O\left(1 / \nu^{1 / 2}\right)(\nu \rightarrow \infty)$, and the second integral equals

$$
\int_{C_{2}} \frac{f(z) d z}{z^{n}(z-1)}-\int_{C_{2}} \frac{f(z) d z}{z^{n+k+1}(z-1)} .
$$

Both of these integrals tend to zero for $n \rightarrow \infty$, which is shown in the same way as (3.4) for $\phi=0$. Hence

$$
\sum_{\nu=n}^{n+k} a_{\nu}=O\left(\sum_{\nu=n}^{n+k} \frac{1}{\nu^{1 / 2}}\right)+o(1)=O\left(\left[\epsilon_{n} n^{1 / 2}\right] \cdot \frac{1}{n^{1 / 2}}\right)+o(1)=o(1) \quad(n \rightarrow \infty),
$$

which proves (4.1) and therefore Theorem 4.

5. Tauberian theorems asserting $E_{p^{-}}$and $S_{\alpha}$-summability. Consider now a power series

$$
f(z)=\sum a_{n} z^{n}
$$

with positive radius of convergence on the boundary of its region of $E_{p^{-}}$and $S_{\alpha}$-summability. We investigate the question from what assumptions about $f(z)$ in the neighborhood of $z=1$ we can derive the summability of $\sum a_{n}$ by means of the methods $E_{p}$ and $S_{\alpha}$. The following assumptions will be frequently used:

(5) Comparing Theorem 4 with Theorem 1 it is noted that the assumptions on $f(z)$ are relaxed in Theorem 4; but since $V-\sum a_{n}=s$ implies $A-\sum a_{n}=s$ if $\lim \sup \left|a_{n}\right|^{1 / n}=1$, the assumptions on $\sum a_{n}$ are strengthened. 


$$
f(z) \text { is regular in } \Omega_{E_{p}}=\Omega\left(\frac{2^{p}-1}{2^{p+1}-1}\right) \quad \text { for } p>0 \text {; }
$$

$\left({ }^{* *}\right) \quad f(z)$ is regular in $|z|<\alpha$ and in $\Omega_{S_{\alpha}}=\Omega\left(\frac{1}{2-\alpha}\right) \quad$ for $0<\alpha<1$.

As to the necessity of these assumptions for the $E_{p^{-}}$and $S_{\alpha^{-}}$-summability of $\sum a_{n}$ see $\$ 2$, a and c.

Corresponding to Theorem 1 we have

Theorem 5. Assume that (5.1) fulfills the condition $\left(^{*}\right)\left[\right.$ or $\left.\left({ }^{* *}\right)\right]$ and is regular and bounded in $S_{2}$, furthermore $E_{p}-\lim a_{n}=0\left[\right.$ or $\left.S_{\alpha}-\lim a_{n}=0\right]$. Then $E_{p}-\sum a_{n}=s$ [or $\left.S_{\alpha}-\sum a_{n}=s\right]$ if $\lim _{z \rightarrow 1-0} f(z)=s$.

Proof. We restrict ourselves to the proof of the case of $E_{p}$-summability. According to $\$ 2$, a the question is whether the series $\sum a_{n}^{\prime} w^{n}$ representing $F(w)=f\left(\phi_{p}(w)\right)$ with $\phi_{p}(w)=w /\left(2^{p}-\left(2^{p}-1\right) w\right)$ converges for $w=1$. We shall show that for $F(w)=\sum a_{n}^{\prime} w^{n}$ all the assumptions of Theorem 1 are fulfilled.

(a) The image of the region $S_{2}$ in the $z$-plane under $w=\phi_{p}^{-1}(z)$ is a region in the w-plane whose boundary has an exterior osculation of order one with $|w|=1$ at $w=1$. Hence $F(w)$ (which by $\left(^{*}\right)$ is regular in $|w|<1$ ) is regular and bounded in some region $S_{2}$ in the w-plane.

(b) We have $a_{n}^{\prime} \rightarrow 0(n \rightarrow \infty)$ since $E_{p}-\lim a_{n}=0(\$ 2, a)$.

(c) Finally $\lim _{w \rightarrow 1-0} F(w)=\lim _{z \rightarrow 1-0} f(z)=s$.

Hence, by Theorem $1, \sum a_{n}^{\prime}=s$, i.e. $E_{p^{-}} \sum a_{n}=s$.

If $f(z)$ is regular at the point $z=1$ on the boundary of the region of summability, Theorem 5 yields analogues to Theorem A of the introduction.

The proof of Theorem 5 is based upon the following idea. Suppose that certain assumptions on $F(w)=\sum a_{n}^{\prime} w^{n}$ allow one to draw conclusions about $\sum a_{n}^{\prime}$, and we "transform" these assumptions on $f(z)$ by $z=\phi_{p}(w)$. Then from the "transformed" assumptions on $f(z)$ we can conclude to $\sum a_{n}^{\prime}$ and therefore to $E_{p^{-}} \sum a_{n}$, since $E_{p^{-}} \sum a_{n}$ behaves like $\sum a_{n}^{\prime}$. Following are two more examples of this general principle.

Theorem 6. Assume that (5.1) fulfills the condition $(*)[$ or $(* *)]$ and is continuous in $\overline{\Omega_{E_{p}}}\left[\right.$ or $\overline{\Omega_{S_{\alpha}}}$. Then $C_{\epsilon} E_{p^{-}} \sum a_{n}\left[\right.$ or $\left.C_{\epsilon} S_{\alpha^{-}} \sum a_{n}\right]$ exists for every $\epsilon>0$.

REMARK. The case where $f(z)$ is regular in $|z|<1$ and continuous in $\overline{\Omega_{E_{p}}}\left[\right.$ or $\overline{\Omega_{S_{\alpha}}}$ ] was treated by Meyer-König [11, p. 352]. He proved that then $E_{p} C_{\epsilon^{-}} \sum a_{n}$ [or $\left.S_{\alpha} C_{\epsilon^{-}} \sum a_{n}\right]$ exists for $\epsilon=1,2, \cdots$. These results are contained in Theorem 6 , for since the matrices $\left(E_{p}\right)$ and $\left(C_{\epsilon}\right)$ are Hausdorff matrices, $\left(C_{\epsilon} E_{p}\right)=\left(E_{p} C_{\epsilon}\right)$; on the other hand it is proved that for $\epsilon=1,2, \cdots$ the methods $C_{\epsilon} S_{\alpha}$ and $S_{\alpha} C_{\epsilon}$ are equivalent [12, p. 450].

Proof of Theorem 6. We restrict ourselves to the case of $E_{p}$-summability. 
The assumptions on $f(z)$ imply that $F(w)=\sum a_{n}{ }^{\prime} w^{n}$ is regular in $|w|<1$ and continuous in $|w| \leqq 1$ whence by a known result of M. Riesz [15, pp. 9495] we obtain that $C_{\epsilon}-\sum a_{n}^{\prime}$ exists for every $\epsilon>0$, i.e. $C_{\epsilon} E_{p^{-}} \sum a_{n}$ exists for every $\epsilon>0$.

If in Theorem 6 the continuity of $f(z)$ for $z \rightarrow 1$ is sufficiently strong, we can derive the $E_{p^{-}}$[or $S_{\alpha^{-}}$] summability of $\sum a_{n}$.

Theorem 7. Assume that $f(z)$ fulfills the condition $\left({ }^{*}\right)\left[\right.$ or $\left.\left({ }^{* *}\right)\right]$ and that

$$
f(z)=s+o\left((1-z)^{\eta}\right)
$$

uniformly for $z \rightarrow 1$ in $\Omega_{E_{p}}\left[\right.$ or $\Omega_{S_{\alpha}}$. Then $E_{p^{-}} \sum a_{n}=s$ [or $\left.S_{\alpha^{-}} \sum a_{n}=s\right]$ if $E_{p}-\lim a_{n}=0$ [or $\left.S_{\alpha}-\lim a_{n}=0\right]$.

The last condition is certainly fulfilled under the stronger assumption that $f(z)$ is continuous in $\overline{\Omega_{E_{p}}}$ [or $\overline{\Omega_{S_{\alpha}}}$ ], since this means that $F(w)$ is continuous in $|w| \leqq 1$, so that $a_{n}^{\prime} \rightarrow 0$, i.e. $E_{p}-\lim a_{n}=0$ [or $S_{\alpha}-\lim a_{n}=0$ ].

Proof. For the function $F(w)=\sum a_{n}^{\prime} w^{n}$ we have

$$
F(w)=f\left(\phi_{p}(w)\right)=s+o\left(\left(1-\phi_{p}(w)\right)^{\eta}\right)=s+o\left((1-w)^{\eta}\right) \quad(\eta>0),
$$

uniformly for $w \rightarrow 1$ in $|w|<1$ since $1-\phi_{p}(w)=\left(2^{p} /\left(2^{p}-\left(2^{p}-1\right) w\right)\right)(1-w)$ $\sim(1-w)$ for $w \rightarrow 1$; furthermore $a_{n}^{\prime} \rightarrow 0$ since $E_{p}-\lim a_{n}=0$. Hence by a known theorem $[17$, p. 220$]$ the series $\sum a_{n}^{\prime}$ converges, i.e. $E_{p^{-}} \sum a_{n}$ exists, and its value is $s$. The proof for the $S_{\alpha}$-case is similar.

Assuming that there exists an analogue to Theorem 7 for $B$-summability (see Theorem $7^{\prime}$, \$6) we obtain the following

COROLlary. Let $f(z)=\sum a_{n} z^{n}$ be regular in $|z|<1$ and

$$
f(z)=s+o((1-z) \eta)
$$

uniformly for $z \rightarrow 1$ in $\Re_{E_{p}}$ [or $\Re_{B}$, or $\Re_{S_{\alpha}}$ ]. Then $E_{p^{-}} \sum a_{n}=s$ [or $B-\sum a_{n}=s$ or $\left.S_{\alpha^{-}} \sum a_{n}=s\right]$.

For the proof one notes that $E_{p}-\lim a_{n}=0$ [or $S_{\alpha}-\lim a_{n}=0$ ] since $a_{n}^{\prime} \rightarrow 0$ is implied by the boundedness of $f(z)$ in $\Omega_{E_{p}}$ [or $\Omega_{S_{\alpha}}$ ]. We shall prove in Theorem 9 that $B$-lim $a_{n}=0$ is a consequence of the boundedness of $f(z)$ in $\Omega_{B}$.

The above corollary has some relationship to a theorem which Hardy and Littlewood have stated without proof $[7$, p. 53]:

Let $f(z)=\sum a_{n} z^{n}$ be regular in $|z|<1$ and $f(z)=s+o\left((1-z)^{\eta}\right) \quad(\eta>0)$, uniformly for $z \rightarrow 1$ in some circle touching $|z|=1$ interiorly at $z=1$. Then $B-\sum a_{n}=s$.

Obviously this result and the corollary overlap, i.e. neither one is included in the other $\left.{ }^{6}\right)$.

We can combine the above corollary with Theorem 3:

${ }^{(6)}$ For a series $\sum a_{n}$ with $\lim \sup \left|a_{n}\right|^{1 / n}=1$ we have $E_{p} \rightarrow B \rightarrow S_{\alpha}$. 
Let $\Omega_{1}$ and $\Omega_{2}$ be two circular regions whose bounding circles touch $|z|=1$ at $z=1$ exteriorly and interiorly, respectively. Assume that $f(z)=\sum a_{n} z^{n}$ is regular in $\Omega_{1}$, continuous in $\overline{\Omega_{1}}$ and strongly continuous for $z \rightarrow 1$ in $\Omega_{2}$ (i.e., $f(z)=s$ $+o\left((1-z)^{\eta}\right)(\eta>0)$, uniformly for $z \rightarrow 1$ in $\left.\Omega_{2}\right)$. Then $\sum a_{n}=s$.

For the proof note that $\Omega_{2}$ is one of the regions of the set $\Omega_{E_{p}}, \Omega_{B}, \Omega_{S_{\alpha}}$.

6. Tauberian theorems asserting $B$-summability. In this last section we shall deal with a power series

$$
f(z)=\sum a_{n} z^{n}
$$

with positive radius of convergence, for which $z=1$ lies on the boundary of its

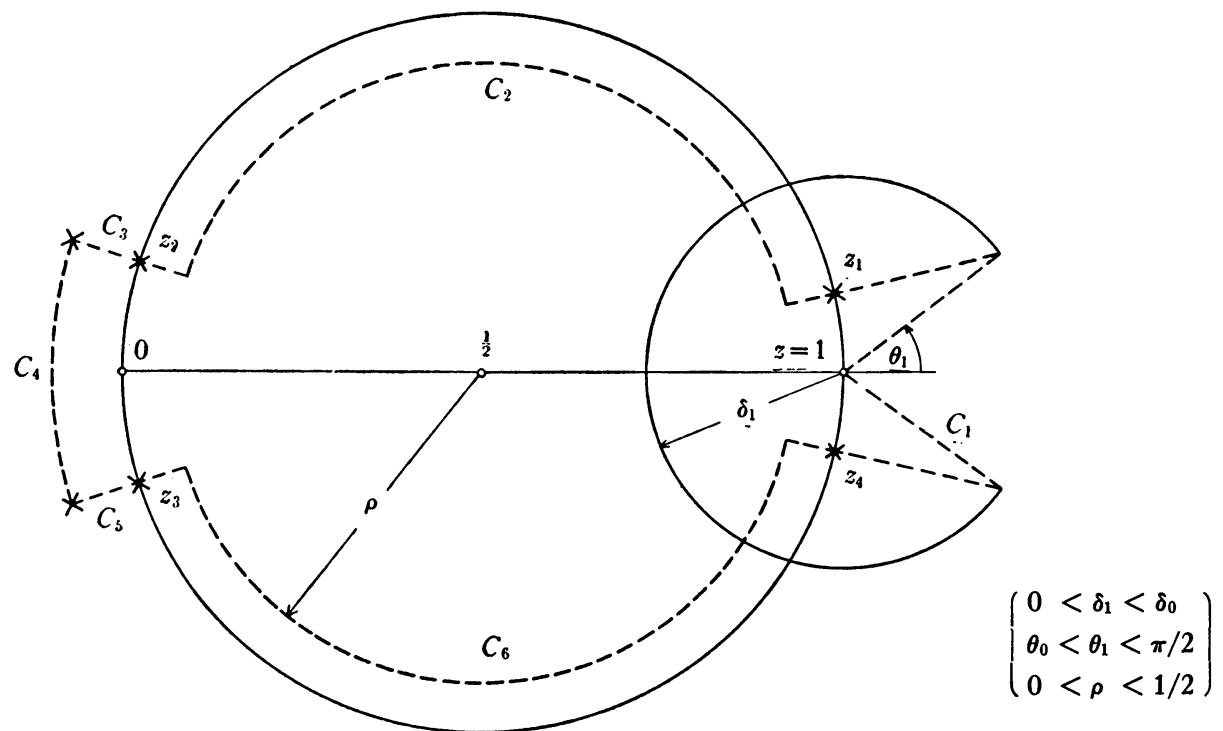

FIG. 2

region of $B$-summability. This implies the regularity of $f(z)$ in $\Omega_{B}$. The section will be divided into three parts. In part $A$ we prove the analogue to Theorem 1, deriving the $B$-summability of $\sum a_{n}$ from assumptions on $f(z)$ in the neighborhood of $z=1$. Also given are sufficient conditions on $f(z)$ which imply $B$-lim $a_{n}=0$. Part B contains a necessary and sufficient condition for the existence of $B-\sum a_{n}$ and analogues to Theorems 6 and 7 are derived. Finally in part $\mathrm{C}$ a theorem of Obrechkoff is discussed.

PART A.

Theorem 8. Let (6.1) be regular and bounded in $S_{2}$, and $B$ - $\lim a_{n}=0$. Then $B-\sum a_{n}=s$ if $\lim _{z \rightarrow 1-0} f(z)=s$.

Proof. Since $z=1$ lies on the boundary of the region of $B^{\prime}$-summability, $B^{\prime}-\sum a_{n} z^{n}$ exists for $0<z<1$, and we have for these values of $z$ 


$$
\begin{aligned}
f(z)=B^{\prime}-\sum a_{n} z^{n} & =\int_{0}^{\infty} e^{-t} \sum \frac{a_{n}(t z)^{n}}{n !} d t=\frac{1}{z} \int_{0}^{\infty} e^{-t / z} \phi(t) d t \\
& =(\zeta+1) \int_{0}^{\infty} e^{-t \zeta} \psi(t) d t=(\zeta+1) I(\zeta) \quad\left(\zeta=\frac{1}{z}-1\right),
\end{aligned}
$$

where we let $\psi(t)=e^{-t} \phi(t)=e^{-t} \sum a_{n}\left(t^{n} / n !\right)=B\left(t ; a_{n}\right)$ for $t \geqq 0$. The integral $I(\zeta)$ exists for $\zeta>0$ and has the limit $s$ for $\zeta \rightarrow 0$, so that by a Tauberian theorem for Laplace-integrals (see for example [6, p. 164]) it is sufficient to show that $\int x \psi(t) d t$ is a slowly oscillating function. We shall show that

$$
\lim _{x \rightarrow \infty} \int_{x}^{x+\epsilon(x) x} \psi(t) d t=0
$$

for any positive function $\epsilon(x)$ tending to zero as $x \rightarrow \infty$. For (6.2) implies that $I(0)=s$, i.e. that $B^{\prime}-\sum a_{n}=s$, which is equivalent to $B-\sum a_{n}=s$ since $B$-lim $a_{n}$ $=0$.

We now prove (6.2). Denote by $C=\sum_{i=1}^{6} C_{i}$ the path as indicated in Fig. 2, and let $|f(z) / z|<M$ for $z$ on $C$. (Let $f(1)=s$; the points $z_{2}$ and $z_{3}$ should be chosen such that $f(z)$ is regular on $C-C_{1}$, thereafter they are fixed.) Then for $t>0$

$$
\begin{aligned}
B\left(t ; a_{n}\right)=\psi(t) & =\frac{1}{2 \pi i} e^{-t} \sum\left(\int_{C} \frac{f(z) d z}{z^{n+1}} \frac{t^{n}}{n !}\right) \\
& =\frac{1}{2 \pi i} e^{-t} \int_{C} \frac{f(z)}{z} \sum \frac{(t / z)^{n}}{n !} d z=\frac{1}{2 \pi i} \int_{C} \frac{f(z)}{z} e^{-t(1-1 / z)} d z,
\end{aligned}
$$

so that

$$
\begin{aligned}
\int_{x}^{x+\epsilon(x) x} \psi(t) d t & =\frac{1}{2 \pi i} \int_{x}^{x+\epsilon(x) x} \int_{C_{1}+\cdots+C_{6}} \frac{f(z)}{z} e^{-t(1-1 / z)} d z d t \\
& =\frac{1}{2 \pi i}\left[I_{1}(x)+\cdots+I_{6}(x)\right] .
\end{aligned}
$$

Divide $C_{1}$ into its four rectilinear segments. Then we have for instance

$$
\left|\int_{1}^{1+\delta_{1} e^{i \theta_{1}}} \frac{f(z)}{z} e^{-t(1-1 / z)} d z\right|<M \int_{0}^{\infty} e^{-\sigma t y} d y=\frac{M}{\sigma t},
$$

since for $z$ on $\left(1,1+\delta_{1} e^{i \theta_{1}}\right)$ we have $\Re(1-1 / z) \geqq \sigma|1-z|$ for a constant $\sigma>0$. With similar estimations for the other parts of $C_{1}$ we obtain

$$
\int_{C .} \frac{f(z)}{z} e^{-t(1-1 / z)} d z=O\left(\frac{1}{t}\right) \quad(t \rightarrow \infty) .
$$

and hence 


$$
I_{1}(x)=\int_{x}^{x+\epsilon(x) x^{\bullet}} O\left(\frac{1}{t}\right) d t=O\left(\frac{1}{x}\right) \cdot \epsilon(x) x=o(1) \quad(x \rightarrow \infty) .
$$

For the estimation of $I_{3}(x)$ and $I_{5}(x)$ note that for instance on $C_{3}$ we have $\Re(1-1 / z) \geqq \tau\left|z_{2}-z\right|$ for a constant $\tau>0$, so that by similar computations as in the case of $I_{1}(x)$ we obtain $\left|I_{3}(x)\right|+\left|I_{5}(x)\right|=o(1)(x \rightarrow \infty)$. For $z$ on $C_{4}$ we have $\Re(1-1 / z) \geqq \eta>0$, so that

$$
\left|\int_{C_{4}} \frac{f(z)}{z} e^{-t(1-1 / z)} d z\right|<2 \pi M e^{-t \eta}=O\left(\frac{1}{t}\right) \quad(t \rightarrow \infty),
$$

whence by the same reasoning as above $I_{4}(x)=o(1)(x \rightarrow \infty)$.

Finally we estimate $I_{2}(x)$ and $I_{6}(x)$.

$$
\begin{aligned}
I_{2}(x) & =\int_{x}^{x+\epsilon(x) x} \int_{C \cdot} \frac{f(z)}{z} e^{-t(1-1 / z)} d z d t=\int_{C_{2}} \int_{x}^{x+\epsilon(x) x} \frac{f(z)}{z} e^{-t(1-1 / z)} d t d z \\
& =\int_{C_{2}} \frac{f(z)}{z-1} e^{-x(1-1 / z)} d z-\int_{C_{2}} \frac{f(z)}{z-1} e^{-x(1+\epsilon(x))(1-1 / z)} d z,
\end{aligned}
$$

and similarly for $I_{6}(x)$. Hence it is sufficient to show that

$$
\lim _{x \rightarrow \infty} \int_{C_{2}+C_{6}} \frac{f(z)}{1-z} e^{-x(1-1 / z)} d z=0 .
$$

For this purpose we choose the constants $b_{1}, b_{2}, b_{3}, b_{4}$ such that

$$
z H(z)=\frac{1}{1-z}+\frac{b_{1}}{z}+\frac{b_{2}}{z} \cdot e^{(1-1 / z)}+\frac{b_{3}}{z} \cdot e^{2(1-1 / z)}+\frac{b_{4}}{z} \cdot e^{3(1-1 / z)}
$$

vanishes at $z_{1}, z_{2}, z_{3}, z_{4}$. Then we get

$$
\begin{aligned}
\int_{C_{2}+C_{6}} z H(z) f(z) e^{-x(1-1 / z)} d z & \\
=\int_{C_{2}+C_{6}} \frac{f(z)}{1-z} e^{-x(1-1 / z)} d z & +b_{1} \int_{C} \frac{f(z)}{z} e^{-x(1-1 / z)} d z+\cdots \\
& +b_{4} \int_{C} \frac{f(z)}{z} e^{-(x-3)(1-1 / z)} d z \\
& -b_{1} \int_{C_{1}+C_{3}+C_{4}+C_{5}} \frac{f(z)}{z} e^{-x(1-1 / z)} d z-\cdots \\
& -b_{4} \int_{C_{1}+C_{3}+C_{4}+C_{*} \cdot} \frac{f(z)}{z} e^{-(x-3)(1-1 / z)} d z
\end{aligned}
$$

Using (6.4) and the corresponding estimations for the paths $C_{3}, C_{4}$, and $C_{5}$, 
we see that the last four terms in (6.6) tend to zero for $x \rightarrow \infty$, while $B$-lim $a_{n}$ $=0$ implies by (6.3) that the second to fifth terms tend to zero for $x \rightarrow \infty$. Hence it remains to show that

$$
\lim _{x \rightarrow \infty} \int_{C_{2}+C_{6}} z H(z) f(z) e^{-x(1-1 / z)} d z=0 .
$$

Now the function $f(z)$ has the representation

$$
f(z)=\frac{1}{z} \int_{0}^{\infty} e^{-t / z} \phi(t) d t \quad\left(z \in \Omega_{B}\right),
$$

where the Laplace-integral converges also at the regular points $z_{1}, z_{2}, z_{3}, z_{4}$ (see [16, pp. 18-19]( $\left.{ }^{7}\right)$ ) and therefore uniformly on $C_{2}+C_{6}$ by a familiar Abelian theorem on Laplace-integrals. The integral in (6.7) becomes therefore

$$
\int_{0}^{\infty} \int_{C_{2}+C_{6}} H(z) e^{(t-x)(1-1 / z)} e^{-t} \phi(t) d z d t \quad=\int_{0}^{\infty} \psi(t) \int_{C_{2}+C_{6}} H(z) e^{(t-x)(1-1 / z)} d z d t
$$

which is an integral-transformation of the function $\psi(t)$ which tends to zero for $t \rightarrow \infty$. This transform tends to zero for $x \rightarrow \infty$ if for any fixed $t_{1}, t_{2}>0$

$$
\lim _{x \rightarrow \infty} \int_{t}^{t_{2}}|c(x, t)| d t=0 \text { and } \limsup _{x \rightarrow \infty} \int_{0}^{\infty}|c(x, t)| d t<\infty,
$$

where

$$
c(x, t)=\int_{C_{2}+C_{6}} H(z) e^{(t-x)(1-1 / z)} d z \quad(t \geqq 0, x \geqq 0) .
$$

Finally the relations (6.8) are proved in the same manner as (3.7) and (3.8) in $\$ 3$, first integrating twice by parts and then estimating $c(x, t)$. This concludes the proof of Theorem 8 .

It should be noted that Theorem 8 combines two theorems analogous to the Theorems A and B of the introduction. We mention the following case.

Corollary. Assume that $z=1$ lies on the boundary of the region of $B$-summability of $f(z)=\sum a_{n} z^{n}$ and that $f(z)$ is regular at $z=1$. Then $B-\sum a_{n}$ exists if $B$ - $\lim a_{n}=0$.

Sometimes it is useful to have " $B-\lim a_{n}=0$ " substituted by an assumption on $f(z)$.

(7) This theorem of M. Riesz states: In $J(w)=\int_{0}^{\infty} e^{-t w} \phi(t) d t$ let $\int_{0}^{x} \phi(t) d t=o\left(e^{x c}\right)(c>0)(x \rightarrow \infty)$ so that $J(w)$ is regular for $\Re(w)>c$. If $J(w)$ is regular at $w_{0}$ with $\Re\left(w_{0}\right)=c$, then $\int_{0}^{\infty} e^{-t w_{0} \phi(t) d t}$ converges. Here we put $w=1 / z, c=1$, and $w_{0}=1$; the assumption $e^{-x} \int_{0}^{x} \phi(t) d t \rightarrow 0(x \rightarrow \infty)$ is clearly fulfilled since $B-\lim a_{n}=0$ and therefore $\phi(t)=o\left(e^{t}\right)(t \rightarrow \infty)$. 
THEOREM 9. Let $f(z)=\sum a_{n} z^{n}$ be regular in $|z|<r(r>0)$ and in $\Omega_{B}$ and assume that $f(z)$ belongs to the class $H^{1}$ of the circle $\Omega_{B}$, i.e. that

$$
\int_{-\pi}^{+\pi}\left|f\left(\frac{1}{2}+R e^{i \theta}\right)\right| d \theta \leqq K \quad \text { for } 0<R<\frac{1}{2} .
$$

Then $B-\lim a_{n}=0$.

Proof. Again we have

$$
2 \pi i B\left(x ; a_{n}\right)=\int_{C} \frac{f(z)}{z} e^{-x(1-1 / z)} d z \quad\left(C=C_{1}+\cdots+C_{4}\right) .
$$

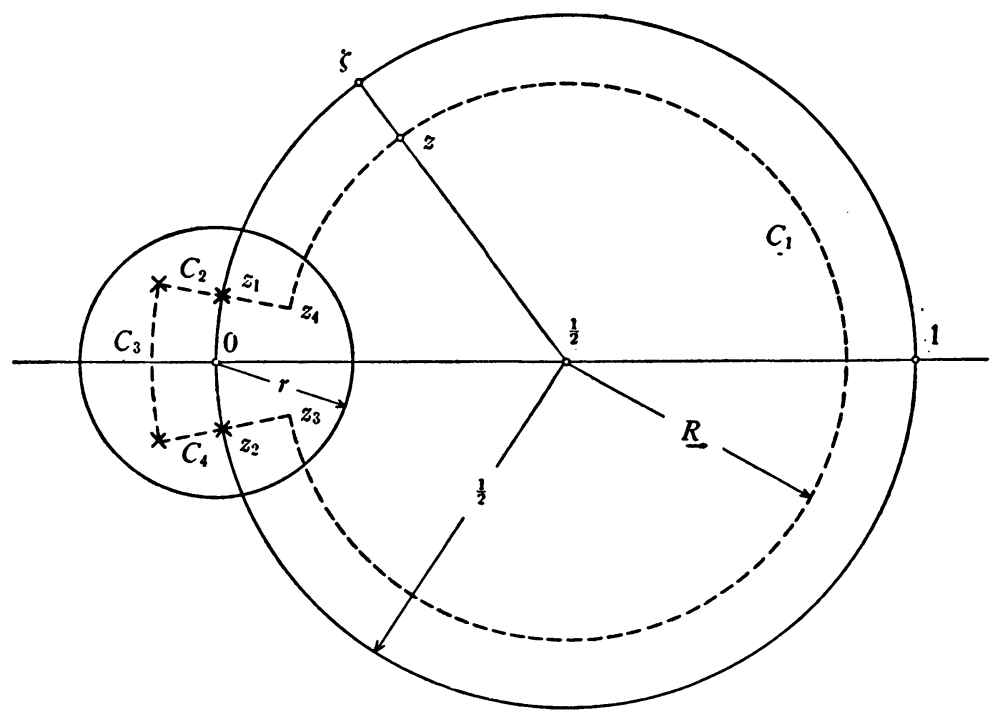

Fig. 3

Given an $\epsilon>0$, we choose $C_{2}$ and $C_{4}$ so short that

$$
\int_{C_{2}+C_{4}}\left|\frac{f(z)}{z}\right||d z|<\frac{\epsilon}{3} \text {. }
$$

Then we have

$$
\left|\int_{C_{2}+C_{4}} \frac{f(z)}{z} e^{-x(1-1 / z)} d z\right| \leqq \int_{C_{2}+C_{4}}\left|\frac{f(z)}{z}\right||d z|<\frac{\epsilon}{3} \quad(x \geqq 0) .
$$

Furthermore

$$
\left|\int_{C_{3}} \frac{f(z)}{z} e^{-x(1-1 / z)} d z\right|=O\left(e^{-x \eta}\right)<\frac{\epsilon}{3} \quad\left(x \geqq x_{1}\right),
$$


since for $z$ on $C_{3}$ we have $\Re(1-1 / z) \geqq \eta>0$. Finally we estimate

$$
I(x)=\int_{C_{1}} \frac{f(z)}{z} e^{-x(1-1 / z)} d z .
$$

Let $x$ be fixed for the moment and put $z(\theta)=1 / 2+R e^{i \theta}, \zeta(\theta)=1 / 2+(1 / 2) e^{i \theta}$, $z_{4}=z(\alpha)$. Then $\lim _{R \rightarrow 1 / 2} f(z(\theta))$ exists for almost all $\theta$ in $(-\pi,+\pi)$ and represents there a Lebesgue-integrable function $f(\zeta(\theta))$ such that

$$
\int_{-\pi}^{+\pi}|f(z(\theta))-f(\zeta(\theta))| d \theta \rightarrow 0 \quad \text { for } R \rightarrow \frac{1}{2}
$$

(see for example $[18$, p. 162]). Therefore in

$$
\begin{aligned}
I(x)= & \int_{-\alpha}^{+\alpha} \frac{f(z(\theta))-f(\zeta(\theta))}{z(\theta)} e^{-x(1-1 / z(\theta))} d z(\theta)+\int_{-\alpha}^{+\alpha} \frac{f(\zeta(\theta))}{z(\theta)} e^{-x(1-1 / z(\theta))} d z(\theta) \\
& +\left(\int_{z_{4}}^{z_{1}}+\int_{z_{2}}^{z_{3}}\right) \frac{f(z)}{z} e^{-x(1-1 / z)} d z
\end{aligned}
$$

the first and third terms tend to zero as $R \rightarrow 1 / 2$, while the second term tends to

$$
\int \frac{f(\zeta)}{\zeta} e^{-x(1-1 / \zeta)} d \zeta
$$

for $R \rightarrow 1 / 2$, the integral being taken as Lebesgue-integral over the arc: $\left(z_{2}, 1, z_{1}\right)$ of $|\zeta-1 / 2|=1 / 2$. Substituting $w=1 / \zeta-1$, we get therefore

$$
I(x)=\int_{-w_{0}}^{+w_{0}} f^{*}(w) e^{w x} d w
$$

where $w$ is purely imaginary and $f^{*}(w)$ is Lebesgue-integrable on the finite section $\left\langle-w_{0},+w_{0}\right\rangle$ of the imaginary axis. By the Riemann-Lebesgue theorem,

$$
|I(x)|<\epsilon / 3 \quad\left(x \geqq x_{2}\right),
$$

which completes the proof of Theorem 9.

It is a well known fact that a power series $f(z)=\sum a_{n} z^{n}$ with radius of convergence 1 is $B$-summable at $z=1$ if $f(z)$ is regular at $z=1$. If we combine Theorems 8 and 9 we obtain the following sharper result:

$A$ power series $f(z)=\sum a_{n} z^{n}$ with radius of convergence 1 is B-summable at $z=1$ if $f(z)$ is regular and bounded in $S_{2}$ and $\lim _{z \rightarrow 1-0} f(z)$ exists.

The result of the author $[5$, p. 331] mentioned previously in $\$ 3$ shows furthermore that herein the region $S_{2}$ cannot be replaced by a region whose boundary has an osculation of second order with $|z|=1$ at $z=1$.

PART B. We give first a necessary and sufficient condition for $B$-summability. 
THEOREM 10. Let $f(z)=\sum a_{n} z^{n}$ be regular in $|z|<r(r>0)$ and in $\Omega_{B}$ and assume that $f(z)$ belongs to the class $H^{1}$ of the circle $\Omega_{B}$, i.e. that (6.9) is fulfilled. Then $B-\sum a_{n}=s$ if and only if

$$
\lim _{x \rightarrow \infty} \frac{1}{\pi} \int_{-\tau}^{+\tau} \frac{\sin x t}{t} f\left(\frac{1}{1+i t}\right) d t=s
$$

for every fixed $\tau>0$, the integral being taken as Lebesgue-integral.

REMARK. Karamata has proved this theorem in the case $r=1$ and under the assumption that $f(z)$ is bounded in $\Omega_{B}$ [8, pp. 156-157], but his method is not applicable under the more general assumptions of Theorem 10.

Proof. We have

$$
2 \pi i B\left(x ; s_{n}\right)=\int_{C_{1}+\cdots+C_{4}} \frac{f(z)}{z(1-z)} e^{-x(1-1 / z)} d z=I_{1}(x)+\cdots+I_{4}(x),
$$

where $C=\sum_{i=1}^{4} C_{i}$ is again the path of Fig. 3. As in the proof to Theorem 9 one finds that for a given $\epsilon>0$

$$
\left|I_{2}(x)\right|+\left|I_{3}(x)\right|+\left|I_{4}(x)\right|<\epsilon / 2 \quad\left(x \geqq x_{1}\right) .
$$

Consider the auxiliary function

$$
H(x)=\int_{C_{1}} \frac{f(z)}{z(1-z)} e^{x(1-1 / z)} d z .
$$

On the segments $\left(z_{4}, z_{1}\right)$ and $\left(z_{2}, z_{3}\right)$ of $C_{1}$ we have $\Re(1-1 / z) \leqq 0$ and therefore

$$
\left|\left(\int_{z_{4}}^{z 1}+\int_{z_{2}}^{z_{3}}\right) \frac{f(z)}{z(1-z)} e^{x(1-1 / z)} d z\right|<\frac{\epsilon}{8} \quad(x \geqq 0)
$$

if only $\left|z_{4}-z_{1}\right|<\delta=\delta(\epsilon)$, and since on the remaining part of $C_{1}$ the estimation $\Re(1-1 / z) \leqq-\eta<0$ holds,

$$
|H(x)|<\epsilon / 4 \quad\left(x \geqq x_{2}\right) .
$$

We now estimate

$$
I_{1}(x)=\int_{C_{1}} \frac{f(z)}{z}\left[\frac{e^{-x(1-1 / z)}-e^{x(1-1 / z)}}{1-z}\right] d z+H(x)=I(x)+H(x)
$$

by a method similar to the one used in the proof of Theorem 9 . The bracketed term is regular for all $z \neq 0$ and therefore $I(x)$ can be treated as $I(x)$ in (6.10). Hence

$$
I(x)=\int \frac{f(\zeta)}{\zeta}\left[\frac{e^{-x(1-1 / \zeta)}-e^{x(1-1 / \zeta)}}{1-\zeta}\right] d \zeta
$$

the integral being taken as Lebesgue-integral along the $\operatorname{arc}:\left(z_{2}, 1, z_{1}\right)$ of 
$|\zeta-1 / 2|=1 / 2$. Letting $\zeta=1 /(1+i t)(t$ real $)$, we get

$$
I(x)=2 i \int_{-T}^{+T} \frac{\sin x t}{t} f\left(\frac{1}{1+i t}\right) d t,
$$

where $z_{2}=1 /(1+i T)$. By the Riemann-Lebesgue theorem,

$$
\left|2\left(\int_{-T}^{-T}+\int_{+\tau}^{+T}\right)\right|<\frac{\epsilon}{4} \quad\left(x \geqq x_{3}\right),
$$

and hence from (6.13)-(6.15) it follows that for $x \geqq \max \left(x_{1}, x_{2}, x_{3}\right)$

$$
\left|2 \pi i B\left(x ; s_{n}\right)-2 i \int_{-\tau}^{+r} \frac{\sin x t}{t} f\left(\frac{1}{1+i t}\right) d t\right|<\epsilon,
$$

which proves Theorem 10.

We prove now the analogues to Theorems 6 and 7 in the case of $B$-summability.

TheOREM $6^{\prime}$. Assume that (6.1) is continuous in $\overline{\Omega_{B}}$. Then $C_{\epsilon} B-\sum a_{n}$ exists for every $\epsilon>0$.

Remark. Since $C_{\epsilon} B$ and $B C_{\epsilon}$ are equivalent methods [2, p. 45], Theorem $6^{\prime}$ contains the result of Karamata [8, p. 157] who proved the $B C_{\epsilon}$-summability of $\sum a_{n}$ under the assumption that $f(z)$ is regular in $|z|<1$ and continuous in $\bar{\Omega}_{B}$.

Proof. For $z$ in $\Omega_{B}$ we have $f(z)=(1 / z) \int_{0}^{\infty} e^{-t / z} \phi(t) d t$, and by a theorem of Riesz $\left[16\right.$, p. 20] the integral is $C_{\epsilon}$-summable for $z=1$, i.e. $C_{\epsilon} B^{\prime}-\sum a_{n}=s$, provided that

$$
\lim _{x \rightarrow \infty} \frac{e^{-x}}{x^{e}} \int_{0}^{x}(x-t)^{\epsilon} \phi(t) d t=0 .
$$

But by Theorem 9 we have $B$-lim $a_{n}=0$, i.e. $\phi(t)=\epsilon(t) \cdot e^{t}$, where $\epsilon(t) \rightarrow 0$ $(t \rightarrow \infty)$. It is easily shown that the integral transformation with the generating function $c(x, t)=e^{t-x} x^{-\epsilon}(x-t)^{\bullet}(0<t \leqq x, \epsilon>0)$ transforms every function tending to zero for $t \rightarrow \infty$ into one tending to zero for $x \rightarrow \infty$, so that (6.16) holds. Now the relation $B\left(x ; s_{n}\right)=B\left(x ; a_{n}\right)+B^{\prime}\left(x ; s_{n}\right)$ implies $C_{\varepsilon} B\left(x ; s_{n}\right)$ $=C_{\epsilon} B\left(x ; a_{n}\right)+C_{\epsilon} B^{\prime}\left(x ; s_{n}\right)$, and the last two terms tend to zero and to $s$ respectively. This proves Theorem $6^{\prime}$.

If the continuity of $f(z)$ for $z \rightarrow 1$ in $\Omega_{B}$ is sufficiently strong, we obtain

Theorem $7^{\prime}$. Assume that (6.1) belongs to the class $H^{1}$ of the circle $\Omega_{B}$, i.e. that (6.9) is fulfilled,

$$
f(z)=s+o\left((1-z)^{\eta}\right) \quad(\eta>0),
$$

uniformly for $z \rightarrow 1$ in $\Omega_{B}$. Then $B-\sum a_{n}=s$. 
Proof. Without loss of generality we may assume $s=0$, so that for the limit function $f(\zeta)$ existing almost everywhere on $|\zeta-1 / 2|=1 / 2$ we have $|f(\zeta)|=|f(1 /(1+i t))| \leqq A|t| \eta$ for $|t|<t_{0}$ where $A>0$ is a constant. Given an $\epsilon>0$, we may choose $\tau<t_{0}$ in (6.12) so small that $\int_{0}^{\tau} t^{t-1} d t<\epsilon \pi / 2 A$. (If (6.12) holds for some $\tau>0$ it holds for all $\tau>0$ by the Riemann-Lebesgue theorem.) For this $\tau$ we obtain

$$
\left|\frac{1}{\pi} \int_{-\tau}^{+\tau} \frac{\sin x t}{t} f\left(\frac{1}{1+i t}\right) d t\right| \leqq \frac{2 A}{\pi} \int_{0}^{\tau} \frac{d t}{t^{1-\eta}}<\epsilon
$$

for all $x \geqq 0$, so that (6.12) is fulfilled.

PART C. This section is concluded with the discussion of a theorem of Obrechkoff [14, p. 1813].

Suppose we have given a power series $f(z)=\sum a_{n} z^{n}$ with positive radius of convergence. Let $z=1$ be a singular point of $f(z)$ lying in the interior of $L$ where $L$ is a rectilinear part of the Borel-polygon associated with $f(z)\left({ }^{8}\right)$. Assume that the singularity of $f(z)$ at $z=1$ is such that in the region $S_{2}$ $=S_{2}\left(\delta_{0}, \theta_{0}\right)$ the function $f(z)$ is regular and $|1-z|{ }^{\delta}|f(z)|$ is bounded for some $\delta(0<\delta<1)$.

The theorem of Obrechkoff states that under these assumptions the series $\sum a_{n} z_{0}^{n}$ is $B$-summable for every regular point $z_{0}=R_{0} e^{i \phi_{0}}$ in the interior of $L$.

Obrechkoff's proof of this theorem is valid only under restricted conditions. If the circle $K:\left|z-z_{0} / 2\right|=R_{1}\left(R_{1}>R_{0} / 2\right)$ is drawn and $A_{1}=1+a_{1} e^{i \theta_{0}}$ and $A_{2}=1+a_{2} e^{-i \theta_{0}}$ are the two points lying on $K$ with $|\operatorname{arc}(z-1)|=\theta_{0}$, his proof depends on the fact that $a_{1}$ and $a_{2}$ tend to zero if $R_{1}$ approaches $R_{0} / 2$. This, however, is true if and only if the circle $\left|z-z_{0} / 2\right|<R_{0} / 2$ is contained in the region $|\operatorname{arc}(z-1)|>\theta_{0}$, i.e. if and only if

$$
\left|\operatorname{arc} z_{0}\right|+\theta_{0} \leqq \pi / 2 \text {. }
$$

Therefore the theorem of Obrechkoff remains valid if either $z_{0}$ is close enough to $z=1$, or if $\theta_{0}$ may be chosen small enough such that (6.17) holds. (Note that $\operatorname{arc}\left(z_{0}-1\right)= \pm \pi / 2$ since $z=1$ is in the interior of $L$; therefore $\left|\operatorname{arc} z_{0}\right|$ $<\pi / 2$.)

But if (6.17) holds, the region $\left|z-z_{0} / 2\right|<R_{0} / 2$ is contained in the region $|\operatorname{arc}(z-1)|>\theta_{0}$. Therefore

$\lim \sup |z-1|^{\delta}|f(z)| \leqq M$ for $z \rightarrow 1$ in $\left|z-\frac{z_{0}}{2}\right|<\frac{R_{0}}{2} \quad(0<\delta<1)$,

whilst $f(z)$ remains bounded for the other part of $\left|z-z_{0} / 2\right|<R_{0} / 2$; this is because $z_{0}$ is an interior point of $L$ and therefore $z=1$ is the only singularity of $f(z)$ on $\left|z-z_{0} / 2\right|=R_{0} / 2$. Theorem 9 assures now that $B \lim a_{n} z_{0}^{n}=0$ and hence by Theorem 8 or its corollary the existence of $B-\sum a_{n} z^{n}$ follows. This

(s)By "interior of $L$ " we mean that $z=1$ is not a corner of the Borel-polygon. 
is a new proof of Obrechkoff's theorem in its modified form.

Added January 28, 1953. It has been investigated by Garten and Karamata [Math. Zeit. vol. 40 (1936) pp. 756-759 and vol. 45 (1939) pp. 635-641] under what conditions on $a_{n}$

$$
\begin{aligned}
B-\sum a_{n}= & s \text { implies } B-\sum \alpha_{n}=s \\
& \left(\alpha_{0}=a_{0}+a_{1}, \alpha_{n}=a_{n+1}, n=1,2, \cdots\right) .
\end{aligned}
$$

Their restrictions on the series $\left[a_{n}=o\left(n^{k}\right)(n \rightarrow \infty, k\right.$ fixed $)$ and $a_{n}=o\left(e^{n^{\rho}}\right)$ $(n \rightarrow \infty, \rho<1 / 3)$, respectively] are such that the associated function $f(z)$ $=\sum a_{n} z^{n}$ is necessarily regular in $|z|<1$. It should be noted that if $f(z)$ does not fulfill this condition, Theorem 9 may be applicable, since $B$ - $\lim a_{n+1}=0$ is necessary and sufficient for the validity of $(6.18)$ (see [6, p. 183]).

\section{BIBLIOGRAPHY}

1. L. Bieberbach, Neuere Untersuchungen über Funktionen von komplexen Variablen, Encyklopädie der mathematischen Wissenschaften, vol. II, 3, 1 (1909-1921) pp. 379-532.

2. G. Doetsch, Eine neue Verallgemeinerung der Borelschen Summabilitätstheorie der divergenten Reihen, Dissertation, Göttingen, 1920. 400.

3. P. Fatou, Séries trigonométriques et séries de Taylor, Acta Math. vol. 30 (1906) pp. 335-

4. D. Gaier, On some Tauberian theorems, Thesis, University of Rochester, N. Y., 1951, 98 pp.

5. - Über die Summierbarkeit beschränkter und stetiger Potenzreihen an der Konvergenzgrenze, Math. Zeit. vol. 56 (1952) pp. 326-334.

6. G. H. Hardy, Divergent series, Oxford, 1949.

7. G. H. Hardy and J. E. Littlewood, Theorems concerning the summability of series by Borel's exponential method, Rend. Circ. Mat. Palermo vol. 41 (1916) pp. 36-53.

8. J. Karamata, Über die B-Limitierbarkeit einer Potenzreihe am Rande, Math. Zeit. vol. 44 (1939) pp. 156-160.

9. E. Landau, Darstellung und Begründung einiger neuerer Ergebnisse der Funktionentheorie, Berlin, 1929.

10. W. Meyer-König, Untersuchungen über einige verwandte Limitierungsverfahren, Math. Zeit. vol. 52 (1949) pp. 257-304. ${ }^{\bullet}$

11. - Die $E_{p^{-}}$und $S_{\alpha^{-}}$Summierbarkeit einer Potenzreihe an der Konvergenzgrenze, Math. Zeit. vol. 52 (1949) pp. 344-354.

12. - Beziehungen zwischen einigen Matrizen der Limitierungstheorie, Math. Zeit. vol. 53 (1951) pp. 450-453.

13. R. Nevanlinna, Eindeutige analytische Funktionen, Berlin, 1936.

14. N. Obrechkoff, Sur la sommation de la série de Taylor sur le contour du polygone de sommabilité par la méthode de M. Borel, C.R. Acad. Sci. Paris vol. 186 (1928) pp. 1813-1815. 89-99.

15. M. Riesz, Über einen Satz des Herrn Fatou, J. Reine Angew. Math. vol. 140 (1911) pp.

16. - Über die Summierbarkeit durch typische Mittel, Acta Litterarum ac Scientiarum Univ. Hungaricae vol. 2 (1924) pp. 18-31.

17. E. C. Titchmarsh, The theory of functions, Oxford, 1950.

18. A. Zygmund, Trigonometrical series, Warsaw, 1935.

UNIVERSITY OF ROCHESTER, ROCHESTER, N. Y. 